\title{
Home visits or telephone contacts may help prevent early childhood caries
}

\author{
Abstracted from \\ Plonka, KA, Pukallus ML, Barnett A, Holcombe TF, Walsh LJ, Seow WK. \\ A controlled, longitudinal study of home visits compared to telephone contacts to prevent early childhood caries. \\ Address for correspondence: W. Kim Seow, Centre for Paediatric Dentistry, School of Dentistry, \\ The University of Queensland, 200 Turbot St,Brisbane, Qld 4000, Australia. E-mail: k.seow@uq.edu.au
}

\section{Question: In newborn children do home visits compared with telephone contacts to mothers reduce early childhood caries when compared with a reference group of children?}

Design Randomisation to intervention groups using Zelen's design with a non-participant community control group recruited at 24 months. Intervention Patients were allocated to either the home visit (HV) group ( $n=236)$ or telephone contact (TC) group $(n=89)$. HVs and TCs were conducted at 6,12 and 18 months. Bacterial tests were given to both groups at baseline and 24 months. The HV group had an additional bacterial test at six months. A community control (reference group) was recruited from the same district at 24 months. All three groups (HV, TC and reference) were examined for caries at 24 months. Outcome measure The primary outcome measure was dental caries (dmft), secondary measures were Streptococcus mutans (MS) and lactobacilli (LB) levels.

Results There was no statistically significant difference in caries between HVs and TCs. However there were statistically significant differences respectively in caries levels between HV/TC and the reference group. There was a statistically significant difference in MS levels between the HV group and both the TC and reference groups, with fewer children with high MS levels in the HV group(28\%) compared with either the TC group(47\%) or reference group (35\%). Conclusions Both HVs and TCs conducted six-monthly from birth are effective in reducing early childhood caries prevalence compared to $\mathrm{RC}$ by 24 months. HVs are also associated with fewer children with MS compared with TC and reference group at 24 months.

\section{Commentary}

Despite reductions in dental disease, caries and in particular early childhood caries remains an important dental public health problem.

Within Scotland the Childsmile Practice programme (www.childsmile.org) has been developed to improve the oral health of children from birth. The programme provides additional support to families with higher risk of oral disease through a programme of home visiting, community initiatives and close relationships with dental practices. Consequently it is of interest to see this Australian study which evaluates the effectiveness of a simple educational programme delivered both through home visits and via the telephone.

Within this study there is a risk of selection bias due to the way the mothers were recruited, in that they were all recruited from community centers. This means that mothers who did not attend community centers were not represented within this study. The reader needs to appreciate that the results of this study may only apply to mothers who attend health clinics, and are not in the more challenging 'hard to reach' groups.

Randomised controlled trials (RCTs) reduce selection bias by ensuring participants have an equal chance of being in each group. One of the problems with conventional RCTs is the inability of participants to choose which intervention they are randomised to. This inability to choose can pose ethical problems (especially if it appears one intervention may be superior to another). This study is interesting in that the authors used Zelen's design ${ }^{1}$ to allow mothers to change their allocation to an intervention depending on their personal preference. The problem with this design is that no matter how appealing the freedom of choice can be, selection bias will increase when people opt to change groups. In this study $9 \%$ of participants opted to change their intervention, and this may in turn have biased the results of this study. Cochrane suggests the use of the intention-to-treat principle(www.cochrane-net.org/openlearning/html/mod14-4.htm); that participants in trials should be analysed in the groups to which they were randomised, regardless of whether they received or adhered to the allocated intervention. The intention-to-treat principle may have been a useful way of overcoming some of the potential disadvantages with Zelen's design.

The reader is advised that the randomisation of participants is in sequential blocks but we are not advised as to the method of randomisation. The importance of outlining the details of both randomisation and allocation is highlighted in the Cochrane handbook(www.cochrane-handbook.org). Such descriptions enable the reader to understand potential areas of bias and also enables the study to be repeated in other areas. 


\section{CARIES}

The authors chose in this instance to use a reference group. The use of a control group as defined by Cochrane may have been a more appropriate choice. Within this study the reader is not informed how the reference group was chosen. One problem with the use of a reference group is that the group of participants may not be chosen from the same population group as the intervention groups. Their characteristics may therefore be dissimilar to the intervention groups, increasing the risk of selection bias.

The authors of the study listed a number of useful characteristics of the mother/child pair that may have acted as confounding factors, eg birth type. The authors state however that there were no demographic differences between the mothers in the three groups. Analysis of the information provided in the paper does however reveal differences in the characteristics of subjects including the percentages of mothers in each group who had their highest level of education as high school, differences in mothers who had dental cavitation at baseline and differences in bacterial levels of mothers in each group. There is evidence to suggest that all these characteristics can increase the risk of decay in children. Berkovitz ${ }^{2}$ highlights the relationship between maternal and infant levels of mutans streptococci. Children of mothers who had high levels of untreated caries have, in one study, been shown to have more than three times the levels of caries experience when compared with children whose mothers had no untreated caries. ${ }^{3}$ Nanjappa highlights in her recent $\mathrm{PhD}$ the relationship between higher education of the mother and the consumption of extrinsic sugars which in turn increase caries risk. ${ }^{4}$

Plonka describes in detail the loss to follow up in participants within this study. This loss was $20.3 \%$ in the HV group, and higher in the TC group (34.8\%). These participants were not able to be followed up due to loss of contact. As CONSORT suggests, such information could perhaps be included in a flow diagram to enable the reader to easily analyse the flow of participants through the study (www.consort-statement.org). The authors should be commended for ensuring the examiners were both blinded and validated in relation to the caries examinations. However, we are not advised whether the examiners who examined the $\mathrm{S}$. mutans tests were also blinded.
The time frame for caries and MS should also be considered. It may be prudent of the researchers to consider a follow-up caries examination when the children are older (and caries prevalence increases) to measure the differences between the three groups.

It is unsure why the researchers chose to perform a six month bacterial test on the HV children when the results of the bacterial test could not be compared with either of the other three groups. Within research it seems ethical to avoid any intervention if there is no benefit to the participant (or researcher).

The authors advise that the examiners checked for white spot lesions and cavitations. Although the reader is advised that there were no white spot lesions in any of the children's examinations it might have been prudent to consider the use of a validated tool such as ICDAS (www.icdas.org) to examine the children. Chesters, for example showed that by using clinical visual diagnostic criteria which included enamel lesions, it was possible to detect differences in the effect of an intervention over a shorter period than when using a system (such as $\mathrm{dmft}$ ) based on caries change extending into dentine. ${ }^{5}$

\section{Practice points}

- There is only weak evidence that home visits are effective at reducing dental decay.

Brett Duane

Department of Public Health, NHS Fife, Cameron Hospital, Windygates, UK.

1. Zelen M. A new design for randomized clinical trials. N Engl / Med. 1979; 300: 1242-1245

2. Berkovitz RJ, Turner I, Green P. Maternal salivary levels of Streptococcus mutans and primary oral infection of infants. Arch Oral Biol. 1981; 26: 147-149

3. Dye BA, Vargas CM, Lee JJ, Magder L, Tinanoff N. Assessing the relationship between children's oral health status and that of their mothers. J Am Dent Assoc. 2011; 142: 173-183

4. Nanjappa S (Supervised by Macenes $W$ and Hector MP) Family functioning and Sugar Consumption in 3 and 4 year old children in outer NE London. Unpublished presentation.

5. Chesters RK, Pitts NB, Matuliene G, et al. An abbreviated caries clinical trial design validated over 24 months. J Dent Res. 2002; 81: 637-640.

Evidence-Based Dentistry (2012) 12, 39-40. doi:10.1038/sj.ebd.6400853 